\title{
Asymmetry in Resting Alpha Activity: Effects of Handedness
}

\author{
Ruth E. Propper ${ }^{1}$, Jenna Pierce ${ }^{2}$, Mark W. Geisler ${ }^{3}$, Stephen D. Christman ${ }^{4}$, Nathan Bellorado² \\ ${ }^{1}$ Psychology Department, Montclair State University, Montclair, USA \\ ${ }^{2}$ Psychology Department, Merrimack College, North Andover, USA \\ ${ }^{3}$ Psychology Department, San Francisco State University, San Francisco, USA \\ ${ }^{4}$ Psychology Department, University of Toledo, Toledo, USA \\ Email: propperr@mail.montclair.edu
}

Received June 16, 2012; revised July 27, 2012; accepted August 12, 2012

\begin{abstract}
Study Aim: Frontal electroencephalographic (EEG) alpha band power during rest shows increased right, and/or decreased left, hemisphere activity under conditions of state or trait withdrawal-associated affect. Non-right-handers (NRH) are more likely to have mental illnesses and dispositions that involve such withdrawal-related affect. The aim of the study was to examine whether NRH might be characterized by increased right, relative to left, hemisphere activity during rest. Methods: The present research investigated that hypothesis by examining resting EEG alpha power in consistently-right-handed (CRH) and NRH individuals. Results: In support of the hypothesis, NRH demonstrated decreased right hemisphere alpha power, and therefore increased right hemisphere activity, during rest, compared to $\mathrm{CRH}$. Conclusions: The study demonstrates further support for an association between increased right hemisphere activity and negative affect via an association between such EEG activity and NRH.
\end{abstract}

Keywords: EEG; Handedness; Asymmetry; Emotion

\section{Introduction}

The approach-withdrawal theory of affect proposes that increased right and/or decreased left hemisphere activity is associated with negative, or withdrawal-related, mood and temperament, including anxiety, sadness, introversion, and in some circumstances anger, while increased left and/or decreased right hemisphere activity is associated with positive, or approach-related affect, including general well-being and happiness. For example, differential hemispheric activation has been specifically associated with depression [1,2], alcoholism [3], anxiety [4,5] self-reported generalized negative affect [6] and under some circumstances, violence potential [7] with these disorders/characteristics characterized by increased right, relative to left, frontal cortical hemisphere activity.

The approach-withdrawal theory has received considerable support from studies measuring hemispheric activation in cortical frontal areas during rest. In particular, alpha band power during rest demonstrates the predicted asymmetries associated with positive (increased left hemisphere activity) versus negative (increased right hemisphere activity) affect or affective style [7-9].

Non-right-handed, relative to right-handed, individuals are more likely to demonstrate dispositional traits and mental illnesses that have been associated with increased right, relative to left, hemisphere, activity, such as aggression [9], worrying [10], anxiety [11], behavioral inhibition [12], depression [13], bipolar disorder [14], and alcoholism and suicide [15].

Given that withdrawal-related affect and some mental illnesses are associated with increased right hemisphere activity, and the non-right-handed are more likely to experience such affect and/or mental illnesses, it is possible that non-right-handers as a group demonstrate increased right, relative to left, hemisphere activity at rest. Interestingly, it may be that it is not direction of hand preference (left versus right) that is related to affect, but rather degree (consistent versus inconsistent regardless of direction) of hand preference. For example, Propper et al. [16], reported significantly greater anger/hostility in inconsistently-handed individuals, compared to the consistently-handed. The inconsistently-handed also reported increased anxiety, depression, and confusion, though these latter differences did not quite reach significance. These findings offer the tantalizing possibility that it is not only direction of hand preference that is related to hemispheric differences in cortical activity, but degree of hand preference as well (see Section 2.2 for additional handedness determination information).

Previous studies have investigated differences in cor- 
tically-derived alpha power as a function of handedness $[17,18]$, however, these studies have been limited to comparisons between left- versus right-handers. Given that degree of hand preference may be as important an individual difference as direction of hand preference on both baseline affect and cortical activity, the current work examines resting hemispheric differences in hemispheric activity in inconsistent- versus consistent-handers. The hypothesis that inconsistently-handed individuals would demonstrate increased right hemisphere activity was examined by comparing resting frontal EEG alpha power in consistently-right- and inconsistently-righthanded individuals. That EEG asymmetries in resting alpha activity may reflect both state and trait characteristics of individuals' moods and emotions suggests the possibility that such a marker could be used to predict susceptibility to some mental illnesses.

\section{Method}

\subsection{Participants}

As part of a previously published protocol [19] 22 undergraduates participated $(\mathrm{M}$ age $=20.11, \mathrm{SD}=1.49)$ for extra credit in a Psychology course. All read and signed an informed consent form, and the experimental protocol received IRB approval from the University of Toledo IRB. A one second, artifact-free epoch from either of the resting baseline conditions was unable to be recovered for two participants; data from these individuals were not included in analyses. 3 additional individuals were eliminated from analyses due to difficulty categorizing their handedness (see below). Final $\mathrm{N}=17$; inconsistent-right-handed $\mathrm{n}=9$; consistent-right-handed $\mathrm{n}$ $=8$ (see below for information on handedness classifications).

\subsection{Hand Preference Determination}

The Edinburgh Handedness Inventory [20] consists of 10 activities self-rated as always, usually, or having no preference of performing with one hand versus the other. The EHI can be scaled from -100 , indicating a perfectly consistent preference for use of the left hand, to +100 , indicating a perfectly consistent preference for right hand use. Thus, handedness can be conceptualized as varying along two dimensions: degree (consistent versus inconsistent) and direction (left versus right), with these two dimensions being represented by different cortical areas [21]. Because our sample contained no consistentlyleft-handed individuals (only about 2\% - 3\% of the population is consistently left-handed [22], making this group most difficult to examine), and only 3 inconsistently- left-handed individuals, and because both degree and direction of hand preference may be related to neuroanatomy and behavior, only those individuals scoring above " 0 " on the EHI were included in analyses. We therefore compared consistently-right-handed (CRH) individuals (handedness scores equal to or above the median value for the sample) with inconsistently-righthanded (IRH) individuals (handedness scores below the sample median). IRH scores ranged from +50 to +85 ( $\mathrm{n}=$ 9, 5 women; $\mathrm{M}=+73.89, \mathrm{SD}=14.31)$; $\mathrm{CRH}$ scores ranged from +90 to $+100(\mathrm{n}=8,5$ women; $\mathrm{M}=+96.88$, $\mathrm{SD}=4.58)$.

\subsection{Materials and Procedure}

Stimuli and instructions were presented on a Macintosh G3 computer with a 16 inch $(40.64 \mathrm{~cm})$ monitor using Superlab Pro 1.75 (Cedrus Corporation, 1997). Participants sat $\sim 60 \mathrm{~cm}$ from the monitor. In the Eyes-Closed condition (EC), participants closed their eyes for $30 \mathrm{sec}-$ onds. In the Eyes-Open condition (EO), participants kept their eyes open and focused on a centrally presented static black dot (approximately 40 of visual angle in diameter) for 30 seconds. Participants performed one EC and one EO block. It should be noted that in Propper et al. (2007) order of EC and EO was counterbalanced between conditions; due to the post-hoc nature of the current investigation, such counterbalancing here was not possible, the result being that $7 \mathrm{CRH}$ had the EO condition first, while 2 IRH had the EO condition first $(\chi=$ $7.24, \mathrm{p}<0.01)$.

\subsection{EEG Materials and Analysis}

Biopac EL503 disposable electrodes were applied using the International 10-20 Electrode Placement System to Fp1 and Fp2, referenced to linked mastoids, and grounded to forehead. These electrode sites were chosen based on the experimental hypotheses in [19]; alpha activity from these sites have also supported the approachwithdrawal hypothesis [23] and are appropriate to examine here. EEG was amplified using the Biopac MP30 (Biopac Systems, Inc., Santa Barbara, CA, USA) and digitally recorded at 200 samples/second on a 12 inch $(30.5 \mathrm{~cm})$ iBook Macintosh Laptop using the Acknowledge 3.6.6 software. Impedance was kept below $10 \mathrm{~K}$ ohms. EEG was inspected for artifact; EEG greater than \pm 200 microvolts was excluded.

The power spectrum for the first artifact-free onesecond epoch per EC and EO Condition was Fast Fourier transformed using a Hamming Window for each participant in the alpha band $(8-13 \mathrm{~Hz})$. This epoch definition was chosen be closer in line with the event-related brain potential literature epochs of 1 second [24,25]. Epochs occurred at least 3 seconds following the beginning or 3 seconds prior to the end of a block. No artifact occurred less than $0.5 \mathrm{sec}$ prior to or following an epoch.

Lateralization Indices (LI) values were calculated via the formula (Left Hemisphere - Right Hemisphere)/(Left 
Hemisphere + Right Hemisphere). Because alpha power is inversely related to hemispheric activity, negative numbers indicate increased left hemisphere alpha power, and a concomitant decrease in left hemisphere, relative to right hemisphere, activity.

\subsection{General Procedure}

Prior to each block, instructions for each condition appeared on the computer screen. The experimenter read aloud these instructions, ensured participants' understanding, and covertly observed the participants' eyes to make sure instructions were followed. EEG was recorded continuously during the experiment. In the EO condition, participants kept eyes focused on the black dot in the center of the computer screen, while in the EC condition participants kept their eyes closed, and were told when to open them. Participants were not asked to think about anything in particular, but merely to "focus on the dot" or to "keep their eyes closed".

\section{Results}

A mixed ANOVA with Handedness (CRH vs IRH) and Hemisphere (Left vs Right) as between- and withinparticipants (respectively) variables was performed on alpha power in the EO and EC conditions separately. A main effect of Hemisphere in the EO condition $(\mathrm{F}(1,1)=$ 4.80, $\mathrm{p}<0.05$; Left hemisphere $\mathrm{M}=-39.81, \mathrm{SD}=3.58$; Right Hemisphere $\mathrm{M}=-41.19, \mathrm{SD}=2.42$ ) was modified by a significant interaction between Handedness and Hemisphere $(\mathrm{F}(1,15)=5.98, \mathrm{p}<0.05)$. Alpha power in IRH was significantly $(p<0.05)$ decreased in the right hemisphere $(\mathrm{M}=-41.57, \mathrm{SD}=2.67)$ compared to the left hemisphere $(\mathrm{M}=-38.83, \mathrm{SD}=4.05)$. $\mathrm{CRH}$ did not differ as a function of hemisphere (Left Hemisphere $\mathrm{M}=-40.92$, $\mathrm{SD}=2.80$; Right Hemisphere $\mathrm{M}=-40.77, \mathrm{SD}=2.21$; See Figure 1). No main effects or interactions were significant in the EC condition ( $p>0.05$ for all comparisons). Unpaired t-tests examining the effects of Handedness on LI in the EO and EC conditions revealed significant differences between $\mathrm{CRH}$ and IRH in the EO condition $(\mathrm{EO} \mathrm{t}(15)=2.36, \mathrm{p}<0.05, \mathrm{CRH} \mathrm{M}=0.001$, $\mathrm{SD}=0.02 ; \mathrm{ICH} \mathrm{M}=-0.04, \mathrm{SD}=0.04)$, with IRH demonstrating decreased left hemisphere alpha power, and increased right hemisphere activity, relative to $\mathrm{CRH}$ (See Figure 2). No handedness effects on LI score occurred in the EC condition $(\mathrm{p}>0.05)$.

\section{Discussion}

To our knowledge, the current paper represents the first report of individual differences as a function of degree of handedness in resting EEG alpha power, with inconsistent right-handedness being associated with decreased alpha power over the right hemisphere (i.e., increased

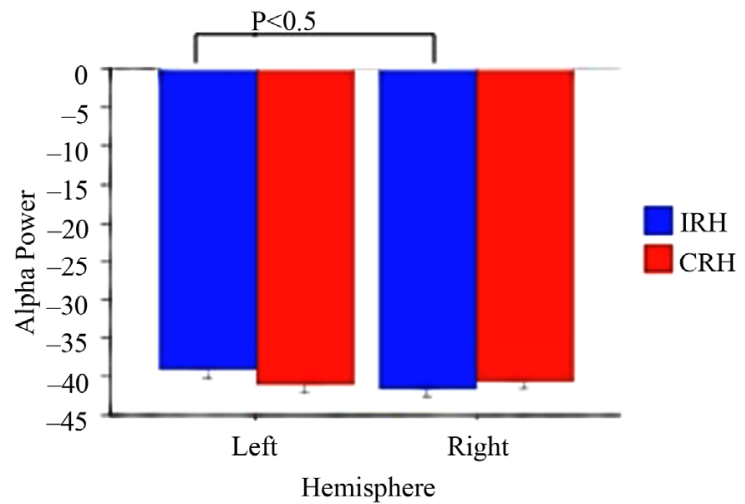

Figure 1. Alpha power as a function of handedness and hemisphere.

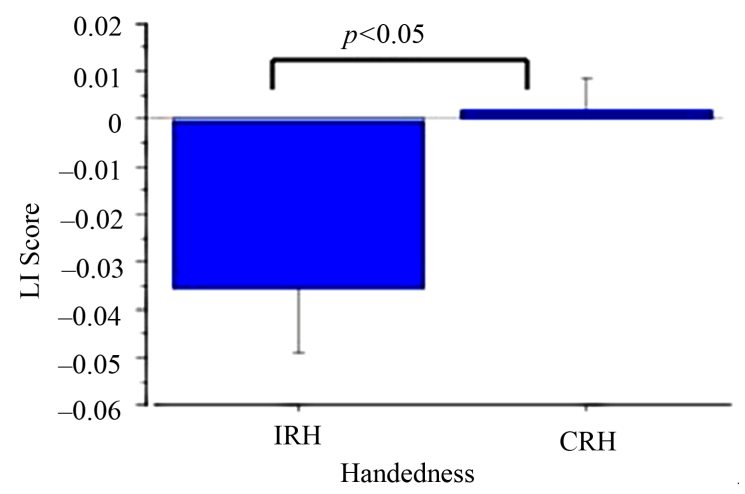

Figure 2. Laterality index score as a function of handedness.

right hemisphere activation). A few prior studies have looked at hemispheric asymmetries in alpha power as a function of direction (i.e.: left versus right) of handedness, finding a similar pattern of increased right hemisphere activation in left-handers $[23,24]$. However, in the present sample we held direction of hand preference constant while examining degree of (right-) handedness, finding a significant difference in resting hemispheric activation. These findings add to a growing literature documenting robust behavioral and physiological differences as a function of degree, rather than direction, of hand preference.

Behaviorally, inconsistent-handedness is associated with superior performance in tasks that may require access to right-hemisphere based processes such as episodic retrieval [26], accurate body image representation [27] and updating of conceptual beliefs [28]. Thus, the current finding of greater relative right hemisphere activation in IRH relative to $\mathrm{CRH}$, in addition to being related to affect, may also manifest in superior performance for IRH across a wide variety of right-hemisphere processes. It is unclear why handedness differences were found only in the eyes open condition or if posterior sites demonstrate handedness differences; these are avenues 
for future exploration.

We would like to point out that the current protocol differs from that typically used to investigate resting EEG alpha; in particular, our examination of only $1 \mathrm{sec}-$ ond epochs may be considered unusual. We find it especially noteworthy that we find handedness differences with this small sample, using small epochs, in this preliminary report our results suggest these handedness difference may be robust. Future research that uses the standard paradigms are indicated.

At the very least, the current results suggest that neuroscientists should consider adding strength of handedness as a variable in their analyses. Even if there is no explicit interest in handedness per se, such inclusion is likely to move variability in data sets from the error term to an effect term, thus enhancing the ability to find significant effects of other variables. At best, the current results suggest that strength of handedness is an important variable of psychological interest. Furthermore, that inconsistent-right-handedness was associated with a pattern of brain activity different from that of the consistent-right-handed indicates that hand preference should be considered in clinical settings.

\section{Conclusion}

The current work suggests that individual differences in degree (consistent versus inconsistent) of hand preference, even among right-handers, is an important variable indicative of resting baseline cortical neural activity. Furthermore, given the link between differential hemispheric activity and affect, the results offer a potential neurophysiological framework for conceptualizing previous findings of an association between non-right- handedness and withdrawal affective states. Ultimately, mechanisms associated with both non-right-handedness and negative affective states may have clinical implica- tions; for example, techniques designed to increased left, or decrease right, hemisphere activity may enable affective modulation. Finally, the current work suggests that degree of handedness should be considered as a possible mediating variable when examining gross brain activity.

\section{Acknowledgements}

Some of the data from this paper were reported at the annual meeting of the Psychonomic Society, Boston, MA (November, 2009).

Parts of this work were supported by a Ciejek Fellowship to R.E.P at Merrimack College. Data was collected at Merrimack College. The authors would like to thank Sean McGraw for assistance with manuscript formatting and for conceptual information in the Introduction.

\section{REFERENCES}

[1] J. Henriques and R. Davidson, "Left Frontal Hypoactivation in Depression," Journal of Abnormal Psychology, Vol. 100, 1991, pp. 535-545. doi:10.1037/0021-843X.100.4.535

[2] C. Schaffer, R. Davidson and C. Saron, "Frontal and Parietal Electroencephalogram Asymmetry in Depressed and Nondepressed Subjects," Biological Psychiatry, Vol. 18, 1983, pp. 753-762.

[3] E. Hayden, R. Wiegand, E. Meyer, L. Bauer, S. O'Connor, J. Nurnberger, D. Chorlian, B. Porjesz and H. Begleiter, "Patterns of Regional Brain Activity in Alcohol-Dependent Subjects," Journal of Alcoholism Clinical Experimental Research, Vol. 30, 2006, pp. 1986-1991. doi:10.1111/j.1530-0277.2006.00244.x

[4] G. Blackhart, J. Minnix and J. Kline, "Can EEG Asymmetry Patterns Predict Future Development of Anxiety and Depression? A Preliminary Study," Biological Psychology, Vol. 72, 2004, pp. 46-50. doi:10.1016/j.biopsycho.2005.06.010

[5] R. Davidson, J. Marshal, A. Tomarken and J. Henriques, "While a Phobic Waits: Regional Brain Electrical and Autonomic Activity in Social Phobics during Anticipation of Public Speaking," Biological Psychiatry, Vol. 47, 2004, pp. 85-95. doi:10.1016/S0006-3223(99)00222-X

[6] R. Davidson, "Emotion and Affective Style: Hemispheric Substrates," Psychological Science, Vol. 3, 2002, pp. 3943. doi:10.1111/j.1467-9280.1992.tb00254.x

[7] R. Davidson, "Well-Being and Affective Style: Neural Substrates and Biobehavioural Correlates," Philosophical Transactions of the Royal Society, Vol. 359, 2004, pp. 1395-1411. doi:10.1098/rstb.2004.1510

[8] H. Urry, J. Nitschke, I. Dolski, D. Jackson, K. Dalton, C. Mueller, M. Rosenkranze, C. Ryff, B. Singer and R. Davidson, "Making a Life Worth Living: Neural Corre- lates of Well-Being," Psychological Science, Vol. 15, 2004, pp. 367-372.doi:10.1111/j.0956-7976.2004.00686.x

[9] S. Dane, and M. Sekertekin, "Differences in Handedness and Scores of Aggressiveness and Interpersonal Relations of Soccer Players," Perceptual and Motor Skills, Vol. 100, 2005, pp. 743-746. doi:10.2466/PMS.100.3.743-746

[10] K. Dillon, "Lateral Preference and Students' Worries: A Correlation," Psychological Reports, Vol. 65, 1989, pp. 496-498. doi:10.2466/pr0.1989.65.2.496

[11] R. Hicks, and R. Pellegrini, "Handedness and Anxiety," Cortex, Vol. 14, 1978, pp. 119-121.

[12] L. Wright, S. Hardie and K. Wilson, "Handedness and Behavioural Inhibition: Left-Handed Females Show Most Inhibition as Measured by BIS/BA Self-Report," Journal of Personality and Individual Differences, Vol. 46, 2008, pp. 20-24. doi:10.1016/j.paid.2008.08.019

[13] K. Denny, "Handedness and Depression: Evidence from a Large Population Survey," Laterality, Vol. 14, 2009, pp. 246-255. doi:10.1080/13576500802362869

[14] O. Fasmer, H. Akiskal, K. Hugdahl and K. Oedegaard, "Non-Right-Handedness Is Associated with Migraine and Soft Bipolarity in Patients with Mood Disorders," Journal of Affective Disorders, Vol. 108, 2008, pp. 217-224. 
doi:10.1016/j.jad.2007.10.028

[15] S. Coren, "The Left-Hander Syndrome: The Causes and Consequences of Left-Handedness," Random House, New York, 2003.

[16] R. E. Propper, T. T. Brunyé, S. D. Christman and J. Bologna, "Negative Emotional Valence Is Associated with Non-Right-Handedness and Increased Imbalance of Hemispheric Activation as Measured by Tympanic Membrane Temperature," Journal of Nervous and Mental Disease, Vol. 198, No. 9, 2010, pp. 691-694. doi:10.1097/NMD.0b013e3181ef1f35

[17] P. Flor-Henry and Z. Koles, "EEG Characteristics of Normal Subjects: A Comparison of Men and Women and of Dextrals and Sinistrals," Research Communications in Psychology, Psychiatry, and Behavior, Vol. 7, 1982, pp. 21-38.

[18] K. O'Connor and J. Shaw, "Field Dependence, Laterality and the EEG," Biological Psychology, Vol. 6, 1978, pp. 93-109. doi:10.1016/0301-0511(78)90049-2

[19] R. Propper, J. Pierce, M. Geisler, S. Christman and N. Bellorado, "Effect of Bilateral Eye Movements on Frontal Interhemispheric Gamma EEG Coherence: Implications for EMDR Therapy," Journal of Nervous and Mental Disease, Vol. 195, 2007, pp. 785-788. doi:10.1097/NMD.0b013e318142cf73

[20] R. Oldfield, "The Assessment and Analysis of Handedness: The Edinburgh Inventory," Neuropsychology, Vol. 9, 1971, pp. 97-113.doi:10.1016/0028-3932(71)90067-4

[21] P. Dassonville, X.-H. Zhu, K. Ugurbil, S. Kim and J. Ashe, "Functional Activation in Motor Cortex Reflects the Direction and the Degree of Handedness," Proceedings of the National Academy of Sciences of the United Stated of America, Vol. 94, 1997, pp. 14015-14018. doi:10.1073/pnas.94.25.14015

[22] L. Lansky, H. Feinstein and J. Peterson, "Demography of Handedness in Two Samples of Randomly Selected Adults $(\mathrm{N}=2083)$," Neuropsychologia, Vol. 26, 1988, pp. 465-477. doi:10.1016/0028-3932(88)90099-1

[23] K. Buss, J. Malmstadt Schumacher, I. Dolski, N. Kalin, H. Goldsmith and R. Davidson, "Right Frontal Brain Activity, Cortisol and Withdrawal Behavior in 6-Month-Old Infants," Journal of Behavioral Neuroscience, Vol. 117, 2003, pp. 11-20. doi:10.1037/0735-7044.117.1.11

[24] J. Intriligator and J. Polich, "On the Relationship between EEG and ERP Variability," International Journal of Psychophysiology, Vol. 20, 1995, pp. 59-74. doi:10.1016/0167-8760(95)00028-Q

[25] K. Spencer and J. Polich, "Post-Stimulus EEG Spectral Analysis and P300-Attention, Task, and Probability," Psychophysiology, Vol. 36, 1999, pp. 220-232. doi:10.1111/1469-8986.3620220

[26] R. Propper, S. Christman and K. Phaneuf, "A MixedHanded Advantage in Episodic Memory: A Possible Role of Interhemispheric Interaction," Journal of Memory and Cognition, Vol. 33, 2005, pp. 751-757. doi:10.3758/BF03195341

[27] S. Christman, M. Bentle and C. Niebauer, "Handedness Differences in Body Image Distortion and Eating Disorder Symptomatology," International Journal of Eating Disorders, Vol. 40, 2007, pp. 247-256. doi:10.1002/eat.20357

[28] S. Christman, B. Henning, A. Geers, R. Propper and C. Niebauer, "Mixed-Handed Persons Are More Easily Persuaded and Are More Gullible: Interhemispheric Interaction and Belief Updating," Laterality, Vol. 13, 2008, pp. 403-426. doi:10.1080/13576500802079646 